\title{
Alfvénic instabilities driven by runaways in fusion plasmas
}

\author{
T. Fülöp ${ }^{1}$ and S. Newton ${ }^{2}$ \\ ${ }^{1}$ Department of Applied Physics, Chalmers University of Technology, Göteborg, Sweden \\ ${ }^{2}$ Euratom/CCFE Fusion Association, Culham Science Centre, Abingdon, Oxfordshire OX14 3DB, \\ United Kingdom
}

(Received 8 May 2014; accepted 14 July 2014; published online 26 August 2014)

Runaway particles can be produced in plasmas with large electric fields. Here, we address the possibility that such runaway ions and electrons excite Alfvénic instabilities. The magnetic perturbation induced by these modes can enhance the loss of runaways. This may have important implications for the runaway electron beam formation in tokamak disruptions. (C) 2014 AIP Publishing LLC.

[http://dx.doi.org/10.1063/1.4894098]

Runaway electron (RE) generation in the presence of electric fields is common in both laboratory and space plasmas. ${ }^{1}$ This can occur because the friction force experienced by an electron due to Coulomb collisions is a non-monotonic function, having a maximum at the thermal speed and decreasing at higher speeds. For sufficiently fast electrons, an accelerating electric force can overcome this friction and the electrons can then run away. In laboratory plasmas, much attention has been given to the highly relativistic RE beams that can be generated in tokamak disruptions. Such REs may damage plasma facing components due to their highly localized energy deposition. The potential for detrimental effects increases with plasma current. Therefore, understanding the processes that may eliminate RE beam formation is very important for future reactor-scale tokamaks with high currents, such as ITER. ${ }^{2}$ In several tokamak experiments, it has been observed that RE generation only occurs above a threshold toroidal magnetic field. ${ }^{3,4}$ While the origin of this threshold is uncertain, it has been linked to decreased relative magnetic fluctuation levels. ${ }^{4,5}$ Recent work at the TEXTOR tokamak ${ }^{6}$ has shown the presence of magnetic fluctuations in the frequency range $f \simeq 60-260 \mathrm{kHz}$, during disruptions deliberately triggered by the injection of argon. The presence of these fluctuations appears to be instrumental in limiting the RE beam formation in these cases. The aim of this work is to investigate low frequency Alfvénic instabilities driven by suprathermal ions and electrons in suddenly cooling impure plasmas and their possible connection with the magnetic fluctuations observed in tokamak disruptions.

There are many observations that Alfvén waves can be driven unstable via particle resonance both in natural and laboratory plasmas. ${ }^{7-9}$ At low frequency, the free energy originates from an inverted energy distribution or spatial inhomogeneity. The resonance condition requires that particles achieve a significant, well defined fraction of the Alfvén velocity $v_{A}=B / \sqrt{\mu_{0} \rho_{m}}$, where $\rho_{m}$ is the mass density. As we will show here, this becomes increasingly difficult as the magnetic field $B$ increases, and could provide a possible explanation for the experimentally observed threshold in the magnetic perturbation level described in Refs. 4-6. Runaway ions are expected to have inverted energy distributions, which will drive Alfvénic instabilities if they attain sufficient velocity to fulfill the resonance condition. Whilst runaway electron energy distributions are not inverted, ${ }^{10}$ Alfvénic instabilities can also be driven by resonant interaction with fast electrons with steep density profiles. Whichever the drive, the appearance of such instabilities in tokamak disruptions can have important consequences. The magnetic perturbations associated with the wave can scatter the runaway electrons and terminate the beam, ${ }^{11}$ providing a passive mitigation of the detrimental effects of the RE beams. Alfvénic instabilities can also be used as a diagnostic for the plasma, through the technique of MHD spectroscopy. ${ }^{12}$

In tokamaks, one of the most important Alfvénic instabilities is the Toroidal Alfvén Eigenmode (TAE). ${ }^{13}$ TAEs are discrete modes residing in toroidicity induced gaps in the shear Alfvén continuum and are therefore usually only weakly damped, as they are not subject to continuum damping. Interestingly, TAEs can have frequencies and mode numbers in the same range as the experimental observations in Ref. 6. TAE modes have been shown to be driven unstable by a wide variety of energetic ion populations, including fast ions produced by neutral beam injection or ion cyclotron resonance heating and alpha-particles produced in DT fusion reactions. ${ }^{7}$ Here, we determine the distribution function of high energy ions in impure, cold plasmas, with large electric fields, characteristic of disruptions. We then consider the conditions under which the runaway ions and electrons can produce TAE growth and discuss the connection with the experimental observations.

Runaway acceleration of ions in the presence of an electric field has been considered during magnetic reconnection events in tokamaks, ${ }^{14}$ solar flares, ${ }^{15}$ and lightning discharges. ${ }^{16}$ To allow ion runaway, the frictional drag due to the drifting electrons should not cancel the electric force. This is the case in the presence of magnetic trapping or impurities with a different charges to that of the ions. ${ }^{17}$ Bulk ions interacting via Coulomb collisions in a plasma experience a non-monotonic friction force. Drag against ions dominates at low energy, decreases with velocity to a minimum at $v_{m}$ and increases at energies exceeding this as drag against electrons takes over. If the ion speed is much lower than the thermal electron speed, $v \ll v_{T e}$, in a straight magnetic field the condition for ions (i) to be accelerated when moving in a Maxwellian distribution of field particles (j) is ${ }^{16} E / E_{D}$ $>\left[\sum_{j}\left(n_{j} Z_{i} Z_{j}^{2} T_{e}\right) /\left(n_{e} T_{j}\right)\left(1+m_{j} / m_{i}\right) G\left(v / v_{T j}\right)\right] /\left|1-Z_{i} / Z_{\text {eff }}\right|$. 
Throughout $m_{s}, T_{s}, n_{s}$, and $Z_{s} e$ denote, respectively, the mass, temperature, number density and charge of particles of species $s, v_{T e}=\sqrt{2 T_{e} / m_{e}}, E_{D}=\left(n_{e} e^{3} \ln \Lambda\right) /\left(4 \pi \epsilon_{0}^{2} T_{e}\right) \quad$ is the Dreicer field, $\ln \Lambda$ is the Coulomb logarithm, $Z_{\text {eff }}=$ $n_{e}^{-1} \sum_{j} n_{j} Z_{j}^{2}$ is the effective charge (where the summation is over all ion species), and $G(x)$ is the Chandrasekhar function. Neutrals are not expected to penetrate the runaway electron beam, ${ }^{10}$ so in this work friction due to collisions with neutral particles will be neglected. The minimum acceleration field $E / E_{D}$ is shown as a function of normalized ion speed in Fig. 1, for deuterium ions in scenarios similar to that described in Ref. 6, where a disruption was triggered by the injection of argon particles. The post disruption temperature is typically in the range of only a few electron volts, although often poorly diagnosed. Whilst the species may be expected to equilibrate under such conditions, we have retained $T_{i} \neq T_{e}$ throughout the analysis, then for numerical evaluations taken a characteristic value of $10 \mathrm{eV}$ for all species. At these low temperatures, the impurities are not fully ionized, and the charge states vary rapidly with time and space. During the disruption itself, the impurities mix into the core and reach high charge states during the cooling phase. They recombine during the current quench but with a possible slight time delay. Here, the heavy argon impurities are taken to have charge $Z_{A r}=2$ or $Z_{A r}=3$, and a typical background carbon impurity with the same charge was also assumed. There is a clear minimum in the collisional friction on the deuterium ions at around $10 \%$ of the electron thermal speed, which is robust to the variation in impurity content. If the electric field is large enough, deuterium ions from the tail of the thermal ion distribution will be accelerated. The instability growth rate will depend on the details of the resulting fast ion distribution, which can be found by solving the kinetic equation for high energy ions.

To allow the study of time-dependent situations, such as a disruptive instability where a steady state is not likely to be established, the kinetic equation must be solved as an initialvalue problem. Such a solution was outlined in Ref. 14 in the limit of trace impurities. Here, we generalise this calculation for arbitrary impurity content. Assuming the friction is

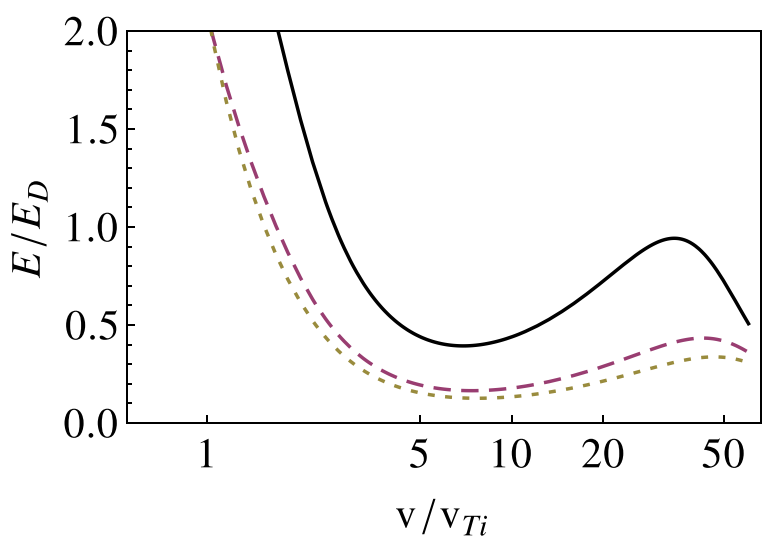

FIG. 1. Minimum accelerating field $E / E_{D}$ as a function of the normalized deuterium ion speed for varying impurity content. Solid: $n_{A r}=0.1 n_{D}$, $n_{C}=0.02 n_{D}, Z_{A r}=Z_{C}=2, Z_{\mathrm{eff}}=1.2$. Dashed: $n_{A r}=0.1 n_{D}, n_{C}=0.08 n_{D}$, $Z_{A r}=Z_{C}=3, Z_{\text {eff }}=1.7$. Dotted $n_{A r}=0.5 n_{D}, n_{C}=0.08 n_{D}, Z_{A r}=Z_{C}=3$, $Z_{\text {eff }}=2.3$. dominated by Coulomb collisions, the kinetic equation for the ion distribution function $f$ can be written as $\partial f / \partial t+$ $v_{\|} \nabla_{\|} f+\boldsymbol{v}_{d} \cdot \nabla f+\left(Z_{i} e E_{*} / m_{i}\right)\left(v_{\|} / v\right)(\partial f / \partial v)=C(f)$, where the subscript $\|$ is taken with respect to the background magnetic field and $\boldsymbol{v}_{d}$ is the magnetic drift due to the field inhomogeneity. When calculating the electron friction force, the distortion of the electron distribution function due to its drift in the imposed electric field has to be taken into account. The drag force on drifting electrons is proportional to the electric field and can be combined with the electric force to give an "effective" electric force $e E_{*}=e E_{\|}+R_{i e \|} / n_{i} Z_{i}$. At the low temperatures of interest, we neglect trapping effects and it can be shown by invoking momentum conservation that $e E_{\|}=-Z_{\text {eff }} R_{i e \|} /\left(n_{i} Z_{i}^{2}\right)$, so that $E_{*}=E_{\|}\left(1-Z_{i} / Z_{\text {eff }}\right)$. Note that in a pure plasma, when $Z_{i}=Z_{\text {eff }}$, the effective field is zero and the test ion will always slow down. The operator $C(f)$ represents collisions between the fast ions and thermal background Maxwellian ions and electrons

$$
\begin{aligned}
C(f)= & \frac{Z_{\mathrm{eff}} v_{c}^{3}}{2 v^{3} \tau_{s}} \frac{\partial}{\partial \xi}\left(1-\xi^{2}\right) \frac{\partial f}{\partial \xi} \\
& +\frac{1}{v^{2} \tau_{s}} \frac{\partial}{\partial v}\left[\left(\bar{n} v_{c}^{3}+v^{3}\right) f+\left(\bar{n} v_{c}^{3}+\frac{T_{e}}{T_{i}} v^{3}\right) \frac{T_{i}}{m_{i} v} \frac{\partial f}{\partial v}\right] .
\end{aligned}
$$

Here $\xi=v_{\|} / v$ is the pitch, the critical velocity for ion slowing on electrons is $v_{c}=\left(3 \sqrt{\pi} m_{e} / 4 m_{i}\right)^{1 / 3} v_{T e}$, the characteristic time for fast ion slowing on electrons $\tau_{s}$ is written in terms of the ion self-collision time $\tau_{i i}=3(2 \pi)^{3 / 2} \epsilon_{0}^{2} \sqrt{m_{i}} T_{i}^{3 / 2} / n_{i} Z_{i}^{4} e^{4} \ln \Lambda$,

$$
\tau_{s}=\left(\frac{m_{i}}{m_{e}}\right)^{1 / 2}\left(\frac{T_{e}}{T_{i}}\right)^{3 / 2} \frac{n_{i} Z_{i}^{2}}{n_{e}} \tau_{i i}, \quad \bar{n}=\sum_{j} \frac{n_{j} Z_{j}^{2} m_{i}}{n_{e} m_{j}},
$$

where the summation is over all ion species. In the trace impurity limit $\bar{n}=Z_{i}$. For the cold plasmas of interest here $Z_{z} m_{i} / m_{z}<1$, so $\bar{n}$ is less than one if the main ions are hydrogenic. For illustration, we note that minimising the dynamic friction, ${ }^{14}$ which is approximately proportional to $\bar{n} v_{c}^{3} / v^{2}$ $+v$ gives $v_{m}=(2 \bar{n})^{1 / 3} v_{c}$, with corresponding kinetic energy $E_{m}=m_{i} v_{m}^{2} / 2=\left(9 \pi m_{i} / 4 m_{e}\right)^{1 / 3} \bar{n}^{2 / 3} T_{e}$. For deuterium ions $E_{m} \simeq 30 \bar{n}^{2 / 3} T_{e}$ and thus they can be accelerated to high energies unless $\bar{n}$ is very low.

In the limit of $\delta=E_{*} T_{i} / Z_{i} E_{D} T_{e} \ll 1$, the solution of the kinetic equation can be obtained by an asymptotic expansion $F=\ln f=\delta^{-1} F^{(0)}+\delta^{-1 / 2} F^{(1)}+\cdots$. Rescaling the independent variables by writing $\tau=3 \delta^{3 / 2}(\pi / 2)^{1 / 2}\left(n_{e} / n_{i} Z_{i}^{2} \tau_{i i}\right) t$ and $w=v\left(\delta m_{i} / T_{i}\right)^{1 / 2}$, the runaway ion distribution function may be given approximately as

$$
\begin{aligned}
f_{R I}(w, \xi, \tau) \propto & \exp \left[-\frac{w^{2}}{2 \delta}+\frac{w^{4}-\left(w^{3}-3 \bar{n} \tau\right)^{4 / 3} H\left(w^{3}-3 \bar{n} \tau\right)}{4 \delta \bar{n}}\right. \\
& +2 w^{2} \sqrt{\left.\frac{2(1+\xi)}{\delta Z_{\text {eff }}}\right]}
\end{aligned}
$$

where $H$ denotes the Heaviside step function. Note that we must restrict to $\delta \ll 1$, therefore the high energy tail of the distribution $f_{R I}$ peaks around $\xi=1$. The solution is valid for small $w$ or short times $\tau \ll 1$, but only holds for $w \leq 1$ when 
$\tau \geq 1$. The distribution $f_{R I}$ reduces to a Maxwellian for $\tau \rightarrow 0$. It must be normalized so that $n_{i}=N(\tau) \int f_{R I} d^{3} v$, where the time-dependent coefficient $N(\tau)$ should reduce to the Maxwellian value as $\tau \rightarrow 0, N(\tau=0)=N_{0}=n_{i} /\left(\sqrt{\pi} v_{T i}\right)^{3}$. By $\tau \sim 1$, the runaway population reaches only a few percent for $\delta \ll 1$, so the time dependence of the normalization constant can be neglected and we take $N(\tau) \simeq N_{0}$.

Figure 2 shows the effect of $\bar{n}$ on the evolution of the leading order piece of the runaway ion distribution (the first two terms in Eq. (2)). In the trace impurity case, the runaway piece of the distribution is well separated from the bulk by the time it reaches significant density. The characteristic velocity $v_{m}$ is much lower at high impurity density and the large electron density decreases the normalized timescale on which the distribution is set up. For typical experimental parameters $\tau_{s}$ is a fraction of a millisecond, so the time to establish the runaway distribution is very short. If, as the runaway ion population builds up, Alfvénic instabilities are excited the analysis leading to the expression $f_{R I}$ will start to break down. Therefore, we consider only the initial phase of the wave-particle interaction and potential instability drive.

The TAE perturbation is typically dominated by two neighbouring toroidally coupled harmonics at large aspect ratio, with poloidal mode numbers $m$ and $m+1 .^{13}$ The mode is localized about the minor radius $r=r_{0}$, at which the magnetic safety factor $q_{0}=(2 m+1) / 2 n$ and has a frequency $\omega=v_{A} /\left(2 q_{0} R_{0}\right)$, where $R_{0}$ is the radius of the magnetic axis. The normalized contribution to the linear growth rate of a TAE from a low collisionality population with distribution $f$ is ${ }^{18,19}$
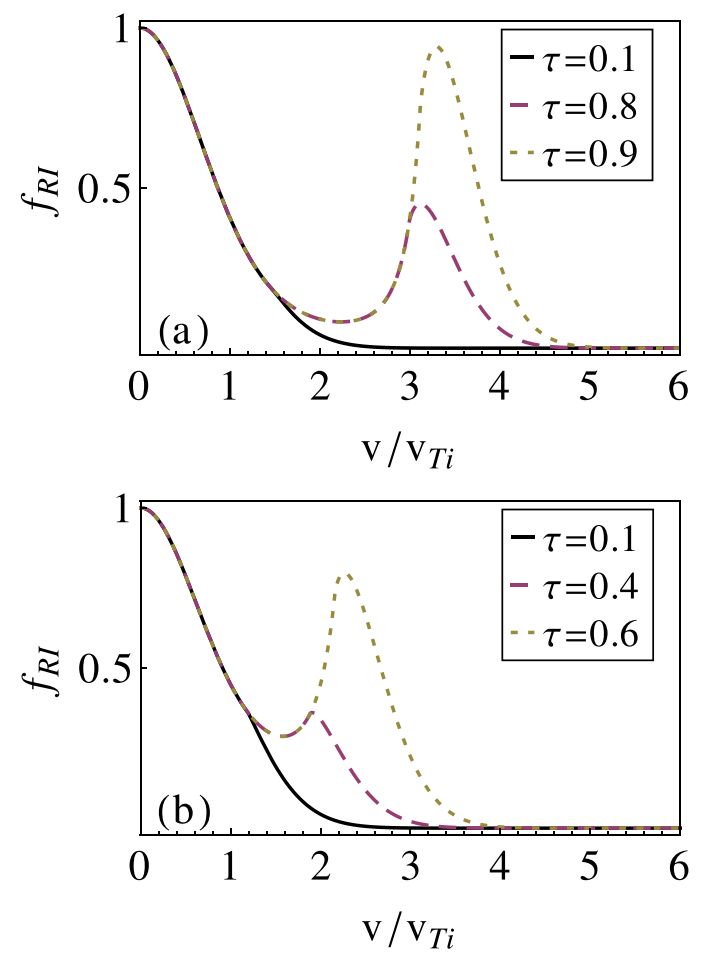

FIG. 2. The leading part of the runaway ion distribution (the first two terms in Eq. (2)) as a function of the deuterium speed normalized to the thermal speed for $\bar{n}=1$ (a) and $\bar{n}=0.5$ (b), where $\delta=0.1$.

$$
\begin{aligned}
\frac{\gamma_{s}}{\omega}= & \frac{2 \pi^{2} \mu_{0} m_{s}^{2} q_{0}^{3} R_{0}}{B_{0}^{2}} \int_{0}^{\infty} d v_{\perp} v_{\perp} \sum_{v_{r}=v_{A}, v_{A} / 3} \frac{v_{r}}{v_{A}} \\
& \times\left.\left(v_{r}^{2}+\frac{v_{\perp}^{2}}{2}\right)^{2}\left(\omega \frac{\partial}{\partial \mathcal{E}}-\frac{n}{q_{s}} \frac{\partial}{\partial \psi}\right) f\right|_{\left|v_{\|}\right|=v_{r}},
\end{aligned}
$$

where $\mathcal{E}=m_{s} v^{2} / 2, \psi$ is the poloidal flux and at large aspect ratio, $d \psi \simeq R B_{\theta} d r$. The two component harmonics allow resonant interaction for $\left|v_{\|}\right| \simeq v_{A} / 3$ or $\left|v_{\|}\right| \simeq v_{A}$, so both runaway ions and electrons, which have opposite velocities, may drive the mode. For brevity, and motivated by the observations of Ref. 6, we specifically consider stability conditions for the case where both $n$ and $\omega$ are positive, but note that alternative combinations can be of interest. ${ }^{20}$

As the runaway ions accelerate, the inverted region of their energy distribution will reach the lower Alfvén resonance and drive the TAE via the $\partial f / \partial \mathcal{E}$ term. In addition, if the radial runaway ion profile peaks on axis, the term proportional to $\partial f / \partial \psi$ will give a positive contribution to the growth rate. For $n_{A r}=0.1 n_{D}=2 \times 10^{18} \mathrm{~m}^{-3}, n_{C}=0.08 n_{D}, B=2 \mathrm{~T}$, and $T_{i}=10 \mathrm{eV}$, the condition $v_{\|}=v_{A} / 3$ requires ions with velocities $v_{R I} \simeq 40 v_{T i}$. For typical low $m \sim 1, q_{0}=1.5$, and $R_{0}=1.75 \mathrm{~m}$, ions with these velocities (or above) would drive a TAE with frequency $112 \mathrm{kHz}$, which is in the frequency range of the observations reported in Ref. 6. Note that a higher amount of assimilated argon or a lower magnetic field would lead to a lower Alfvén velocity and TAE frequency, and in that case the resonance condition with runaway ions would be more readily met. Figure 3 shows the normalized deuterium speed that is required to fulfill the resonance $v_{\|}=v_{A} / 3$ for various temperatures.

At low temperatures, the analytical form of the distribution given in Eq. (2) breaks down before the ions accelerate sufficiently and the growth rate must be calculated using a numerical solution of the ion kinetic equation. However, we give an illustrative analytical calculation of the runaway drive for $T_{e}=500 \mathrm{eV}$, with $\delta=0.1, Z_{A r}=2$, and $Z_{C}=6$. The electric field builds up as the plasma temperature is quenched in the disruption, and so it is also possible that such a resonant interaction can begin during the cooling process. The gradient with respect to energy is related to that with respect to $w$ as $\partial f_{R I} / \partial \mathcal{E}=\left(2 \delta / m_{i} v_{T i}^{2} w\right)\left(\partial f_{R I} / \partial w\right)$. Restricting the runaway density to a few percent allows gradients arising

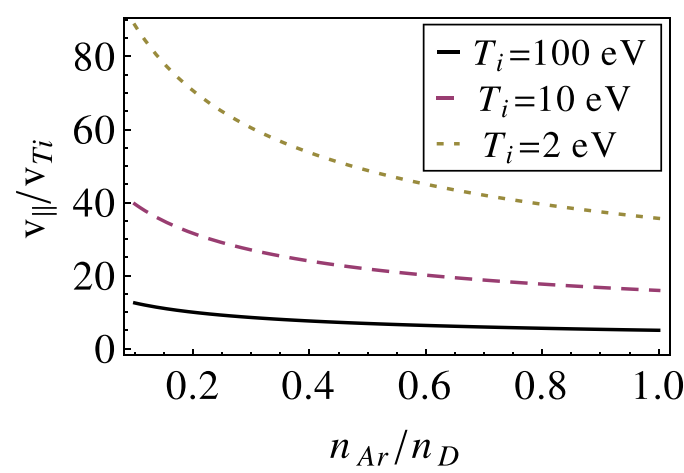

FIG. 3. Normalized deuterium ion speed satisfying the $v_{\|}=v_{A} / 3$ resonance as a function of the assimilated argon content for $B=2 \mathrm{~T}, n_{D}=2 \times 10^{19} \mathrm{~m}^{-3}$, and $n_{C}=0.08 n_{D}$. 
from the normalization constant to be neglected, and taking $\xi \approx 1$ at the resonance, only the term in $v_{r}^{5}$ contributes in Eq. (3). At $\tau=2.27$, the runaway density is $1.2 \%$ of the initial bulk ion density and the normalized drive at the $v_{A} / 3$ resonance is $1.5 \%$. Note that although fast ions resulting from fusion reactions or auxiliary heating often excite highfrequency instabilities (magnetosonic or ion cyclotron waves), ion runaways are not likely to reach high enough velocities to become resonant with higher frequencies and excite such waves.

Finally, we consider the runaway electron population. Anisotropy in pitch-angle is known to drive instability, ${ }^{21}$ but this will not be effective for the low frequency modes of interest here. From Eq. (3) however, we see that TAE modes can also be driven by resonant interaction with fast electrons with steep density profiles. Such profiles may arise at radially localized current sheets, which are often formed due to a thermal instability resulting from a balance between heat-diffusion, radiation, and Ohmic heating. ${ }^{22}$ The characteristic radial scale of the current sheets according to the numerical simulations in Ref. 23 are in the subcentimeter range. In hot plasmas, typically $v_{A}<v_{T e}$ and the bulk electrons produce damping. However, in cold plasmas the condition $v_{A}>v_{T e}$ becomes relevant. Then, we may expect energy transfer to the mode from the well populated lower energy part of the runaway electron distribution. We speculate that kinetic corrections to the TAE mode structure $^{24,25}$ may allow interaction to occur with the electron population over a range of energies in this region. This would give rise to a distribution in the observed magnetic fluctuation frequencies and so may be of relevance in understanding the similar observations in TEXTOR. ${ }^{6}$ Such an interaction may be expected to be of less relevance in the case of runaway ion drive, due to the very localised bump-on-tail form of their distribution.

The higher the magnetic field, the higher the Alfvén velocity, so fewer REs will be able to fulfill the resonance. This reduces the growth rate and may be the explanation for the disappearance of the fluctuations at high magnetic fields. Note that the situation is complicated by the fact that the Alfvén velocity is heavily influenced by the impurity content. Experimental observations at JET show that traces of runaways can be found for argon massive gas injection even at magnetic fields down to $1.2 \mathrm{~T}^{2}$, whilst for neon injection much weaker runaway signatures are observed. This may indicate that the Alfvén speed remains near the electron thermal speed in the presence of the heavier impurity, preventing resonance, and allowing the runaway beam to form.

If the electron runaway generation is dominated by primary generation, the suprathermal part of the electron distribution can be approximated by $f_{e}^{\mathrm{RE}}\left(w_{e \|}, w_{e \perp}\right) \simeq C_{e}\left(\ln w_{\| e}\right)^{-1}$ $\exp \left\{-w_{\perp}^{2} /\left[2\left(1+Z_{\text {eff }}\right) \ln w_{\| e}\right]\right\}$, in the nonrelativistic limit. ${ }^{26}$ Here, $w=v(2 E)^{1 / 2} / v_{T e}, E=\left|E_{\|}\right| / E_{D}$, the normalization constant is determined by $n_{r e}=\int f_{e}^{R E} d^{3} v$ to be $C_{e}=n_{r e} E /$ $\left(\pi v_{e, \max } v_{T e}^{2}\left(1+Z_{\mathrm{eff}}\right)\right), n_{r e}$ is the runaway electron density and $v_{e, \max }$ is the time-dependent maximum parallel velocity of the runaway electrons (which will be close to the speed of light). Taking only the $v_{r}=v_{A}$ resonance, the TAE growth rate due to the spatial inhomogeneity can be rewritten as

$$
\frac{\gamma_{e}}{\omega}=\left.\frac{v_{A}}{\left|\omega_{c e}\right|} \frac{2 \pi^{2} n q_{0}^{4}}{\epsilon_{0}} \frac{m_{e}}{m_{i}} \frac{v_{A} v_{T e}^{2}}{n_{0}} \int d x_{\perp} x_{\perp}\left(1+\frac{x_{\perp}^{2}}{2} \frac{v_{T e}^{2}}{v_{A}^{2}}\right)^{2} \frac{\partial f_{e}}{\partial r}\right|_{v_{\|}=v_{A}}
$$

where $\omega_{c e}$ is the electron cyclotron frequency, $\epsilon_{0}=r_{0} / R_{0}, x$ is the velocity normalized to $v_{T e}$, and $n_{0}=\rho_{m} / m_{i}$. This may be evaluated approximately using $f_{e}^{R E}$, by assuming that the spatial variation of the runaway distribution dominates any variation in the background plasma, which gives, with $w_{\| A}=v_{A}(2 E)^{1 / 2} / v_{T e}$,

$$
\begin{aligned}
\frac{\gamma_{e}}{\omega}[\%]= & \frac{v_{A}}{\left|\omega_{c e}\right|} \frac{\pi n q_{0}^{4}}{\epsilon_{0} \bar{v}_{e, \text { max }}} \frac{m_{e}}{m_{i}}\left(1+\frac{v_{T e}^{2}}{v_{A}^{2}} \frac{\left(1+Z_{\mathrm{eff}}\right) \ln w_{\| A}}{E}\right. \\
& \left.+\frac{v_{T e}^{4}}{2 v_{A}^{4}} \frac{\left(1+Z_{\mathrm{eff}}\right)^{2}\left(\ln w_{\| A}\right)^{2}}{E^{2}}\right) \frac{n_{r e, 17}}{n_{0,19}} \frac{1}{L_{n}^{p}},
\end{aligned}
$$

where $n_{0,19}$ and $n_{r e, 17}$ are the bulk and fast electron densities expressed in units of $10^{19} \mathrm{~m}^{-3}$ and $10^{17} \mathrm{~m}^{-3}$, respectively, $\bar{v}_{e, \text { max }}=v_{e, \text { max }} / v_{A}$ and $1 / L_{n}^{p}=\left[\partial_{r} n_{r e} / n_{r e}-\partial_{r} \bar{v}_{e, \text { max }} / \bar{v}_{e, \max }\right]$. The radial derivative of the maximum velocity is expected to have opposite sign to the radial variation of the runaway density, so enhances the instability growth rate in the case of destabilization. In the event of thermal instability, when radially localized current sheets are formed, the runaway electron density gradient is very steep and the term corresponding to the variation of the maximum energy is negligible in comparison. If secondary generation dominates, the distribution function from Ref. 21 can be used and the growth rate becomes

$$
\frac{\gamma_{e}}{\omega}[\%]=\frac{v_{A}}{\left|\omega_{c e}\right|} \frac{\pi n q_{0}^{4} v_{A}}{\epsilon_{0} c_{z} c} \frac{m_{e}}{m_{i}} e^{-\frac{v_{A}}{c c_{Z}}}\left(1+\frac{2 c}{\alpha v_{A}}+\frac{2 c^{2}}{\alpha^{2} v_{A}^{2}}\right) \frac{n_{r e, 17}}{n_{0,19}} \frac{1}{L_{n}^{s}},
$$

where $\alpha=(\hat{E}-1) /\left(1+Z_{\text {eff }}\right), \hat{E}=E m_{e} c^{2} / T_{e}, c_{z}=\sqrt{3\left(Z_{\text {eff }}+5\right) /}$ $\pi \ln \Lambda$ and $1 / L_{n}^{s}=\partial_{r} n_{r e} / n_{r e}$. The growth rate is expected to be significant at radial locations with large safety factors $q_{0}$ and short spatial gradient scale lengths.

Any drive will be countered by various damping mechanisms. The remaining bulk ions and electrons will be collisional at the low temperatures considered, so their contribution to the damping cannot be evaluated from Eq. (3) and would have to be determined by a collisional treatment of the bulk species. In the case of runaway electron driven TAE, the energy gradient is also expected to damp the wave and Alfvénic instabilities are then only destabilized if the effect of the spatial gradient is dominant. Also, as the parameter profiles evolve during the thermal quench and post-disruption, we may expect that continuum damping of the $\operatorname{mode}^{27}$ can arise and to calculate this would require simulation of the detailed TAE structure. Such damping calculations are beyond the scope of this work.

In this paper, we have considered general forms of the distributions of runaway particles, which have the potential to excite low frequency Alfvénic instabilities. With regard to the observations of spontaneous magnetic fluctuations appearing in fusion plasma disruptions, we find that a variety 
of mechanisms allow TAE modes to be driven unstable by runaways. A steep spatial gradient of runaway electrons is effective in low temperature plasmas, such as those typical of post-disruption conditions in tokamaks, whilst runaway ions form an inverted energy distribution and can give an effective drive in impure plasmas at higher temperatures $(\gtrsim$ $100 \mathrm{eV}$ ). In both cases, the drive is most efficient for low magnetic fields and can therefore account for the absence of instability at higher magnetic fields. The magnetic perturbation associated with the instability is expected to scatter the runaway electrons and in certain cases may therefore stop beam formation. Observation of the excited waves can also be used for diagnostic purposes.

The authors are grateful to J. Connor, A. Stahl, J. Hastie, P. Helander, E. Hollmann, and G. Papp for fruitful discussions.

This project has received funding from the European Union's Horizon 2020 research and innovation programme under grant agreement number 633053, and the RCUK Energy Programme (under grant EP/I501045). The views and opinions expressed herein do not necessarily reflect those of the European Commision.

${ }^{1}$ R. M. Kulsrud, Y.-C. Sun, N. K. Winsor, and H. A. Fallon, Phys. Rev. Lett. 31, 690 (1973).

${ }^{2}$ M. Lehnen, A. Alonso, G. Arnoux, N. Baumgarten, S. A. Bozhenkov, S. Brezinsek, M. Brix, T. Eich, S. N. Gerasimov, A. Huber, S. Jachmich, U. Kruezi, P. D. Morgan, V. V. Plyusnin, C. Reux, V. Riccardo, G. Sergienko, M. F. Stamp, and JET EFDA Contributors, Nucl. Fusion 51, 123010 (2011).

${ }^{3}$ R. D. Gill, B. Alper, M. de Baar, T. C. Hender, M. F. Johnson, V. Riccardo, and Contributors to the EFDA-JET Workprogramme, Nucl. Fusion 42, 1039 (2002).

${ }^{4}$ R. Yoshino, S. Tokuda, and Y. Kawano, Nucl. Fusion 39, 151 (1999).

${ }^{5}$ T. Kudyakov, S. S. Abdullaev, S. A. Bozhenkov, K. H. Finken, M. W. Jakubowski, M. Lehnen, G. Sewell, O. Willi, and Y. Xu, Nucl. Fusion 52, 023025 (2012).
${ }^{6}$ L. Zeng, H. R. Koslowski, Y. Liang, A. Lvovskiy, M. Lehnen, D. Nicolai, J. Pearson, M. Rack, H. Jaegers, K. H. Finken, K. Wongrach, Y. Xu, and the TEXTOR Team, Phys. Rev. Lett. 110, 235003 (2013).

${ }^{7}$ W. W. Heidbrink, Phys. Plasmas 15, 055501 (2008).

${ }^{8}$ W. J. Hughes, D. J. Southwood, and B. Mauk, Nature 275, 43 (1978).

${ }^{9}$ F. Zonca, P. Buratti, A. Cardinali, L. Chen, J.-Q. Dong, Y.-X. Long, A. V. Milovanov, F. Romanelli, P. Smeulders, L. Wang, Z.-T. Wang, C. Castaldo, R. Cesario, E. Giovannozzi, M. Marinucci, and V. Pericoli Ridolfini, Nucl. Fusion 47, 1588 (2007).

${ }^{10}$ E. M. Hollmann, M. E. Austin, J. A. Boedo, N. H. Brooks, N. Commaux, N. W. Eidietis, D. A. Humphreys, V. A. Izzo, A. N. James, T. C. Jernigan, A. Loarte, J. Martin-Solis, R. A. Moyer, J. M. Munoz-Burgos, P. B. Parks, D. K. Rudakov, E. J. Strait, C. Tsui, M. A. Van Zeeland, J. C. Wesley, and J. H. Yu, Nucl. Fusion 53, 083004 (2013).

${ }^{11}$ P. Helander, L.-G. Eriksson, and F. Andersson, Phys. Plasmas 7, 4106 (2000).

${ }^{12}$ H. A. Holties, A. Fasoli, J. P. Goedbloed, G. T. A. Huysmans, and W. Kerner, Phys. Plasmas 4, 709 (1997).

${ }^{13}$ C. Z. Cheng and M. S. Chance, Phys. Fluids 29, 3695 (1986).

${ }^{14}$ P. Helander, L.-G. Eriksson, R. J. Akers, C. Byrom, C. G. Gimblett, and M. R. Tournianski, Phys. Rev. Lett. 89, 235002 (2002).

${ }^{15}$ G. D. Holman, Astrophys. J. 452, 451 (1995).

${ }^{16}$ T. Fülöp and M. Landreman, Phys. Rev. Lett. 111, 015006 (2013).

${ }^{17}$ H. P. Furth and P. H. Rutherford, Phys. Rev. Lett. 28, 545 (1972).

${ }^{18}$ R. Betti and J. P. Freidberg, Phys. Fluids B 4, 1465 (1992).

${ }^{19}$ T. Fülöp, M. Lisak, Ya. I. Kolesnichenko, and D. Anderson, Plasma Phys. Controlled Fusion 38, 811 (1996).

${ }^{20}$ P. Sandquist, S. E. Sharapov, M. Lisak, and T. Johnson, Phys. Plasmas 14, 122506 (2007).

${ }^{21}$ T. Fülöp, G. Pokol, P. Helander, and M. Lisak, Phys. Plasmas 13, 062506 (2006).

${ }^{22}$ S. Putvinski, N. Fujisawa, D. Post, N. Putvinskaya, M. N. Rosenbluth, and J. Wesley, J. Nucl. Mater. 241-243, 316 (1997).

${ }^{23}$ T. Fehér, H. M. Smith, T. Fülöp, and K. Gál, Plasma Phys. Controlled Fusion 53, 035014 (2011).

${ }^{24}$ H. L. Berk, R. R. Mett, and D. M. Lindberg, Phys. Fluids B 5, 3969 (1993).

${ }^{25}$ A. Fasoli, J. B. Lister, S. E. Sharapov, D. Borba, N. Deliyanakis, C. Gormezano, J. Jacquinot, A. Jaun, H. A. Holties, G. T. A. Huysmans, W. Kerner, J.-M. Moret, and L. Villard, Phys. Rev. Lett. 76, 1067 (1996).

${ }^{26}$ R. Cohen, Phys. Fluids 19, 239 (1976).

${ }^{27}$ H. L. Berk, J. W. Van Dam, Z. Guo, and D. M. Lindberg, Phys. Fluids B 4, 1806 (1992). 